\title{
CARACTERIZAÇÃO DAS CADEIAS CURTAS DE ABASTECIMENTO DE ALIMENTOS
}

\section{Jéssica Magero Canellas ${ }^{1}$, Camila Elisa dos Santos Alves ${ }^{1}$}

${ }^{1}$ Doutorandas do Programa de Pós Graduação em Agronegócios da Universidade Federal do Rio Grande do Sul, Porto Alegre, Brasil (camilaelisaalves@gmail.com)

Recebido em: 08/04/2017 - Aprovado em: 10/06/2017 - Publicado em: 20/06/2017 DOI: 10.18677/EnciBio_2017A26

\begin{abstract}
RESUMO
Cadeias curtas de abastecimento têm sido focos centrais de um grande número de pesquisas. Formas alternativas de agricultura e abastecimento de alimentos sustentam essa reorganização da cadeia. Entretanto, conceitos apresentados carecem de definições sobre o tema. Desse modo, o presente estudo teve como objetivo identificar e compreender como se caracterizam as cadeias curtas de abastecimento e quais são as suas abrangências e delimitações. Para tanto, foi realizada uma análise bibliométrica, por meio de uma busca estratégica nas bases de dados Web of Science, Cab direct e Scopus. A análise revelou que as cadeias curtas de abastecimento apresentam abordagens sócio econômicas, estruturais e organizacionais, bem como, aspectos de sustentabilidade, saúde e segurança alimentar.
\end{abstract}

PALAVRAS-CHAVE: circuitos curtos; produtores rurais; venda direta.

\section{CHARACTERIZATION OF SHORT FOOD SUPPLY CHAINS}

\begin{abstract}
Short food supply chains have been central focus of many surveys. Alternative forms of agriculture and food supply support this reorganization of the chain. However, presented concepts lack definitions about the theme. Thus, the present study aims to identify and understand how short food supply chains are characterized and what their scope and delimitations are. For this, a bibliometric analysis was carried out, through a strategic search in the Web of Science, Cab direct and Scopus databases. The analysis reveal that short food supply chains presents socio-economic, structural and organizational approaches, as well as aspects of sustainability, health and food safety.
\end{abstract}

KEYWORDS: Short circuits; farmers; direct sale. 


\section{INTRODUÇÃO}

A produção de alimentos no mundo tem gerado avaliações críticas sobre quando e como atender a produção e a demanda mundial por alimentos. Uma série de indagações e procura por alternativas viáveis tem sido foco de pesquisas. Em um sistema cada vez mais globalizado, foi observado que apenas uma diminuição acentuada do consumo per capita e uso de recursos sustentáveis poderiam contribuir para um mundo mais equitativo (ROCKSTRÖM et al., 2009). Dessa forma, estudos alternativos sobre padrões de produção alimentar têm demonstrado uma tendência para uma produção de alimentos mais regional e local (RENTING et al., 2003; DONALD et al., 2010). Esses sistemas são conhecidos como local foods. A produção, o processamento e o varejo de alimentos são realizados dentro de uma área geográfica delimitada, proporcionando benefícios e impactos socioeconômicos e ambientais desejáveis (KIRWAN, 2004).

Construído como uma ideia de colaboração para moldar economias alimentares autossuficientes, os local foods integram a produção, transformação, distribuição e consumo, com o objetivo de melhorar o bem-estar (saúde econômica, ambiental e social) de uma determinada localidade (FEENSTRA, 2002). Ademais, estes locais são considerados um meio promissor de apoiar o desenvolvimento rural e transformar as relações produtores/consumidores. Por meio do processamento e venda local, uma proporção crescente de valor é agregado e retido pelos agricultores, podendo contribuir também para o emprego local e regeneração econômica de uma região (KIRWAN, 2004). Um dos principais benefícios do sistema local foods é o incentivo a relações próximas entre produtores e consumidores de alimentos, além de promover um modo mais sustentável de produção e consumo. Os local foods dependem de uma organização coletiva e de relações sociais próximas entre produtores e consumidores (HOLLOWAY et al., 2006).

A proliferação de diversas iniciativas para desenvolver estes sistemas locais tem gerado desafios quanto à sua delimitação e entendimento claro dos objetivos. Existem várias tentativas na literatura para categorizar abordagens e concepções que, muitas vezes, se sobrepõem, conhecidas como "alternative", "local", "local markets", e, ainda "short food supply chain", ou, cadeias curtas de abastecimento de alimentos. As conceituações baseiam-se principalmente nas relações entre agricultores e consumidores (FORSSELL \& LANKOSKI, 2015), criando um contato mais direto com o usuário final do produto. Ao mesmo tempo, uma localidade ganha significado social e espacial, criando especificidade e singularidade (MARSDEN et al., 2000).

De maneira geral, as cadeias agroalimentares têm sido caracterizadas em longas ou curtas, as quais incorporam um conjunto de características específicas. Cadeias alimentares longas são descritas como aquelas que abastecem grandes redes de supermercados em áreas urbanas e regionais. Além do varejo, envolvem transportes interestaduais com produção mínima para entrega de produtos e são fortemente regulamentados pela indústria e governo (MIGLIORE et al., 2015).

As cadeias curtas operam, em sua grande maioria, de forma independente e incluem comunidades e cooperativas agrícolas de pequena e média produção. Características têm sido propostas para diferenciar as cadeias longas e curtas de abastecimento de alimentos, como a distância física entre produtores e consumidores; número de intermediários, os atores ao longo da cadeia e a natureza dos relacionamentos (redes e compartilhamento de informações entre os atores) (ILBERY \& MAYE, 2005; HOLLOWAY et al., 2006; CROWE \& SMITH, 2012). Já 
SONNINO \& MARSDEN (2006), distinguem cadeias curtas e longas em termos de escala espacial (local, regional e nacional), a relação com o Estado (novas inovações na governança estão associadas com cadeias curtas), os valores de produção de alimentos (além de valor de commodities), e de nível de rendimentos e impactos na produção.

Simplificar a distinção entre cadeias curtas e longas pode não ser suficiente. O termo cadeias curtas de abastecimento foi implantado para descrever cadeias de fornecimento alternativas ou locais, começando com a ideia de curtas distâncias físicas entre produtores e consumidores e caracterizadas com poucos ou sem intermediários entre produtores e consumidores (CROWE \& SMITH, 2012).

Dessa forma, existe uma necessidade de conceitos mais específicos que ajudem a entender as cadeias curtas de abastecimento. O presente estudo teve como objetivo identificar e compreender como se caracterizam as cadeias curtas de abastecimento e quais são as suas abrangências e delimitações. Em vista disso, este trabalho pode ser visto em parte como uma contribuição para a compreensão da estruturação das cadeias curtas de abastecimento, bem como, seus principais aspectos.

\section{MATERIAIS E MÉTODOS}

A fim de compreender as noções existentes sobre cadeias curtas de abastecimento, foi proposta uma análise bibliométrica. Esta forma de análise é utilizada para examinar a estrutura de conhecimento e o desenvolvimento de campos de pesquisa com base na análise de publicações relacionadas. A pesquisa foi realizada nas bases de dados online Web of Science, Cab direct e Scopus, considerando população, intervenção e resultados buscados. Para a atribuição da amostra, foi utilizado o termo "SHORT* FOOD SUPPLY CHAIN".

Para a seleção dos artigos, foram realizadas avaliações do título, resumo e palavras-chave, utilizando como critérios iniciais o caráter científico do artigo; o idioma (somente artigos publicados na língua inglesa entraram para a seleção), e a presença de investigação da temática de cadeias curtas de abastecimento.

Por meio da pesquisa, foram encontrados 55 artigos, dos quais nove estavam duplicados e 15 foram rejeitados por não se enquadrarem nos critérios de avaliação estabelecidos. Posteriormente, cinco artigos foram rejeitados, pois os mesmos não estavam disponíveis para acesso em bancos de dados. Os 26 artigos restantes foram lidos, de forma que oito foram eliminados por não se enquadrarem na temática desejada (Figura 1). Após, os 31 estudos foram buscados na forma íntegra e avaliados. Destes, 18 foram pertinentes e incluídos na revisão. 


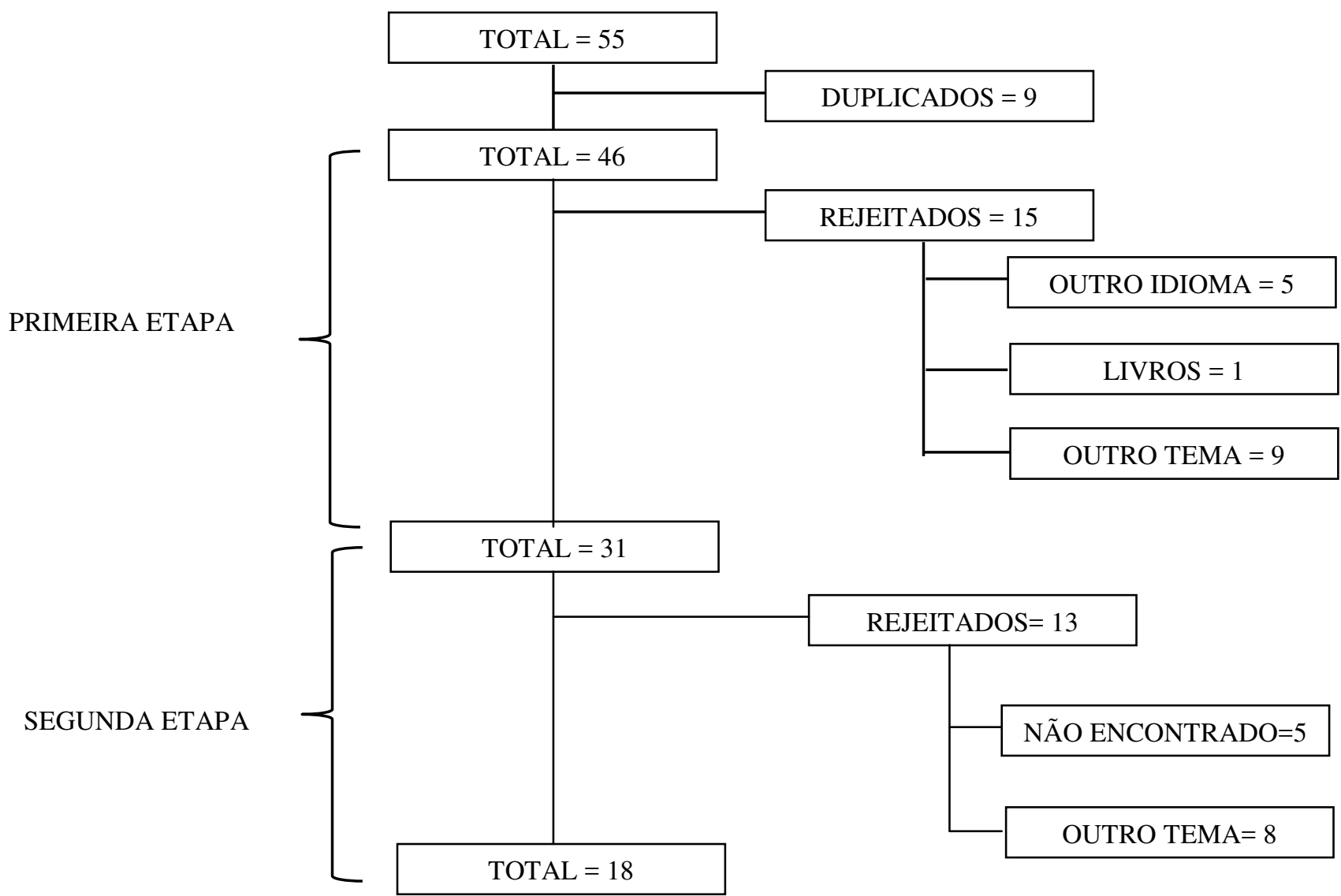

FIGURA 1. Fluxograma da seleção de artigos

Fonte: elaborado pelas autoras, (2016).

\section{RESULTADOS E DISCUSSÃO}

A estratégia de busca foi elaborada com o intuito de fornecer uma pesquisa abrangente e específica, contendo artigos (pesquisas primárias e revisões bibliográficas) que abordassem o tema cadeias curtas de abastecimento de alimentos. Diversos aspectos foram encontrados, relacionados direto ou indiretamente ao tema (quadro 1).

QUADRO 1. Dados e tipos de abordagens utilizadas pelos artigos encontrados

\begin{tabular}{|c|c|c|c|c|}
\hline Autor & País & Ano & Estudo & Abordagem \\
\hline Tudisca et al & Itália & 2015 & Short supply food chain & $\begin{array}{lll}\text { Aspectos } & \text { sociais } & \text { e } \\
\text { econômicos } & & \end{array}$ \\
\hline Smith et al & Austrália & 2015 & Short supply food chain & $\begin{array}{l}\text { Resiliência e segurança dos } \\
\text { alimentos na cadeia }\end{array}$ \\
\hline $\begin{array}{l}\text { Rogers } \\
\text { Fraszczak }\end{array}$ & França & 2014 & $\begin{array}{l}\text { Direct supply food } \\
\text { chain }\end{array}$ & Aspectos sociais \\
\hline Mastronardi et al & Itália & 2015 & Short supply food chain & $\begin{array}{l}\text { Aspectos estruturais, } \\
\text { econômicos e sustentáveis. }\end{array}$ \\
\hline Haas et al & $\begin{array}{l}\text { Estados } \\
\text { Unidos }\end{array}$ & 2014 & Local food & $\begin{array}{l}\text { Percepção e associação à } \\
\text { cadeia }\end{array}$ \\
\hline Borec \& Prisenk & Slovênia & 2013 & Local food & $\begin{array}{l}\text { Aspectos organizacionais e } \\
\text { de cooperação }\end{array}$ \\
\hline Bimbo et al & Itália & 2015 & Short supply food chain & Benefício à saúde \\
\hline
\end{tabular}

ENCICLOPÉDIA BIOSFERA, Centro Científico Conhecer - Goiânia, v.14 n.25; p. 282 


\begin{tabular}{|c|c|c|c|c|}
\hline Balázs & Hungria & 2012 & Local food & $\begin{array}{l}\text { Sustentabilidade e percepção } \\
\text { à cadeia }\end{array}$ \\
\hline Aubry \& Kebir & França & 2013 & Short supply food chain & $\begin{array}{lr}\text { Preservação } & \mathrm{e} \\
\text { desenvolvimento } & \mathrm{da} \\
\text { agricultura urbana } & \end{array}$ \\
\hline Ross & $\begin{array}{l}\text { Estados } \\
\text { Unidos }\end{array}$ & 2005 & Short food supply chain & Aspectos estruturais e sociais \\
\hline $\begin{array}{l}\text { Renting, } \\
\text { Marsden } \quad \text { \& } \\
\text { Banks }\end{array}$ & $\begin{array}{l}\text { Países } \\
\text { Baixos }\end{array}$ & 2003 & Short food supply chain & $\begin{array}{l}\text { Aspectos estruturais, sociais } \\
\text { e econômicos }\end{array}$ \\
\hline Ilbery \& Maye & $\begin{array}{l}\text { Escócia- } \\
\text { Inglaterra }\end{array}$ & 2005 & Short food supply chain & $\begin{array}{l}\text { Aspectos estruturais } \\
\text { sustentabilidade da cadeia }\end{array}$ \\
\hline $\begin{array}{l}\text { Marsden, Banks } \\
\text { \& Bristow }\end{array}$ & Reino Unido & 2000 & Short food supply chain & $\begin{array}{l}\text { Aspectos estruturais, sociais } \\
\text { e econômicos }\end{array}$ \\
\hline Belliveau & Canadá & 2005 & Local food & $\begin{array}{l}\text { Aspectos estruturais, sociais } \\
\text { e econômicos }\end{array}$ \\
\hline Lehtinen & Finlândia & 2012 & Local food & Sustentabilidade da cadeia \\
\hline $\begin{array}{l}\text { Wubben, Fondse } \\
\text { \& Pascucci }\end{array}$ & Holanda & 2013 & Short food supply chain & $\begin{array}{lll}\begin{array}{l}\text { Aspectos } \\
\text { sociais }\end{array} & \text { econômicos } & \text { e } \\
\end{array}$ \\
\hline Ilbery et al & Reino Unido & 2004 & Short food supply chain & $\begin{array}{ll}\text { Aspectos } & \text { sociais } \\
\text { econômicos } & \\
\end{array}$ \\
\hline $\begin{array}{l}\text { Levidow } \\
\text { Psarikidou }\end{array}$ & Reino Unido & 2011 & Short food supply chain & $\begin{array}{ll}\begin{array}{l}\text { Aspetos } \\
\text { econômicos }\end{array} & \text { sociais } \\
\end{array}$ \\
\hline
\end{tabular}

Fonte: elaborado pelas autoras, (2016).

Os aspectos sociais e econômicos foram tratados em mais da metade dos estudos selecionados. Nestes trabalhos, são apresentados os atores que compõem a cadeia e como os fatores sociais influenciam no desenvolvimento. De acordo com BALÁZS et al. (2012), as vendas diretas podem reconfigurar as relações entre produtores e consumidores, assumindo uma característica de justiça social, incentivando relações mais harmoniosas da comunidade e uma participação mais democrática dos participantes na cadeia de abastecimento de alimentos. Igualmente, questões econômicas são expostas para que a viabilidade financeira seja avaliada, refletindo nos aspectos organizacionais da mesma. Da mesma forma, aspectos estruturais e organizacionais são abordados, pois englobam 0 relacionamento em torno da cadeia e como os alicerces são formados.

Segundo MASTRONARDI et al. (2015), agricultores participantes das cadeias curtas de abastecimento podem obter rendas significativas com a venda direta de produtos, gerando maior controle sobre a produção e demanda local, diminuindo custos com excedentes de produção. As vendas diretas permitem que os consumidores obtenham alimentos frescos e saudáveis, com preços mais razoáveis, além de fomentar a sustentabilidade ecológica (TUDISCA et al., 2015). Também, aos consumidores, a mesma forma de mercado pode ser lucrativa, pois o valor necessário para a obtenção do alimento é reduzido, podendo ser utilizado em outros mercados, gerando assim, desenvolvimento e crescimento regional.

Para ROGERS \& FRASZCZAK (2014), os produtores podem estar tecnicamente envolvidos em vendas diretas ou de cadeia curta, mas na prática a relação comercial é normalmente mediada. Os autores defendem que a diferenciação entre vendas diretas e indiretas, ou seja, com ou sem intermediários, facilitaria 0 entendimento na abordagem para diferenciação das cadeias. Ainda, segundo estes autores, existe certa confusão entre o número de transações econômicas com o número de interações sociais que acompanham o produto agrícola em seu caminho do agricultor ao consumidor final. Algumas características 
distintivas são usadas para definir as cadeias curtas de abastecimento de alimentos: proximidade geográfica; viabilidade econômica; interação social; e sustentabilidade ambiental (WUBBEN et al., 2013).

A sustentabilidade relacionada as cadeias curtas de produção e abastecimento de alimentos é tema recorrente em todos os artigos investigados e é apontada como uma das maiores vantagens relacionadas ao tema. Este aspecto é favorecido por um aumento geral do interesse público em questões como ecologia, saúde e bem-estar.

Aspectos relacionados à saúde, segurança alimentar, bem como a percepção dos consumidores em relação as cadeias curtas de abastecimento foram pontuais nos artigos avaliados. De acordo com BIMBO et al. (2015), a participação de local farms em cadeias curtas de abastecimento parece ter, além de repercussões sociais benéficas, potencial impacto positivo sobre as dietas dos consumidores, por meio de aumento no consumo de produtos orgânicos, levando a um aumento no consumo de alimentos saudáveis, como frutas e verduras, resultando em uma população mais bem nutrida.

A capacidade de vincular produtores e consumidores está no cerne destas iniciativas (LEHTINEN, 2012). Segundo MARSDEN et al. (2000), existem basicamente três tipos de cadeias curtas: face a face, de proximidade e as expandidas. A cadeia curta face a face caracteriza-se pela venda direta de quem produz para quem consome. Os consumidores compram produtos diretamente do produtor e a autenticidade e a confiança são medidas pela interação pessoal. Nesta categoria, características específicas do local de produção ou do processo de produção são parâmetros essenciais para definir a qualidade do produto (MARSDEN et al., 2000; RENTING et al., 2003).

O segundo tipo caracteriza-se pela proximidade espacial, ou seja, os produtos são produzidos e distribuídos em uma região específica de produção, e os consumidores estão cientes da natureza local do produto (MARSDEN et al., 2000). $\mathrm{Na}$ cadeia curta expandida, o consumidor está consciente de como foi produzido o alimento e tem confiança em consumi-lo (HUGHES \& ISENGILDINA-MASSA, 2015). Neste caso, os produtos são vendidos fora da região de produção e a distância entre o produtor e o consumidor não é considerada. O valor do produto está associado e pode ser transmitido por meio da identificação da região de produção (MARSDEN et al., 2000; BELLIVEAU, 2005).

Para MARSDEN et al. (2000), a criticidade das cadeias curtas de abastecimento de alimentos não está necessariamente no número de vezes que um produto é manipulado ou na distância ao longo da qual este é transportado, mas no fato do produto chegar ao consumidor incorporado com informações em embalagens impressas ou comunicadas pessoalmente no ponto de venda, isto é, o ponto central para a existência de uma cadeia curta não está no fator de proximidade geográfica, mas na informação.

As distâncias percorridas pelo produto dentro do mesmo Estado foram definidas como cadeias locais, mas ainda há uma falta de consenso sobre o que constitui cadeias de suprimento geograficamente curtas (LIM \& HU, 2016). De acordo com SMITH et al. (2015), na Austrália, onde as distâncias entre produtores e consumidores podem ser grandes, produtos rotulados como "feito na Austrália" não são vistos como locais para muitos consumidores, enfatizando que características como segurança e viabilidade de abastecimento são aspectos importantes, independentemente do conceito aplicado. 
Do ponto de vista do consumidor, os contatos próximos com os produtores e as informações obtidas por meio de relacionamentos, agregam valor ao produto, constroem confiança na qualidade do produto e geram apoio para o produtor (ROSS, 2005). Esta análise revelou a existência de diversas iniciativas e formas de apresentação de cadeias curtas de abastecimento. Inicialmente marginalizadas, essas cadeias de abastecimento parecem ser parte de uma transformação evolutiva do sistema agroalimentar local. Esta tendência está se desenvolvendo em uma linha de demanda por produtos saudáveis, sustentáveis e confiáveis.

\section{CONCLUSÃO}

A tentativa de desenhar um panorama de publicações relacionadas com as cadeias curtas de abastecimento mostrou a divergência de conceitos sobre o tema. Nesta pesquisa foram fornecidas novas pistas sobre a composição das cadeias curtas de abastecimento de alimentos, em comparação com características encontradas na literatura.

O estudo da literatura e da investigação empírica auxiliou no esclarecimento das abordagens presentes dentro desta temática, as quais apresentam aspectos estruturais, sócio econômicos e sustentáveis, da mesma maneira que apontam considerações sobre saúde e segurança alimentar.

Em suma, as cadeias, para serem consideradas como curtas na literatura, requerem proximidade geográfica, participação ativa do produtor e consumidor, bem como, disponibilidade de informação sobre 0 produto que está sendo comercializado.

\section{REFERÊNCIAS}

AUBRY, C.; KEBIR, L. Shortening food supply chains: A means for maintaining agriculture close to urban areas? The case of the French metropolitan area of Paris. Food Policy, v. 41, p. 85-93, 2013. Disponível em: <http://dx.doi.org/10.1016/j.foodpol.2013.04.006>. doi: 1016/j.foodpol.2013.04.006

BALÁZS, B. Local food system development in Hungary. International Journal of Sociology of Agriculture and Food, v.19, n.3, p. 403-421, 2012. Disponível em: <http://dx.doi.org/0798-1759/ ijsaf.org>. doi: 0798-1759/ ijsaf.org

BELLIVEAU, S. Resisting glogal, buying local: Goldschimidt revisited. The Great Lakes Geographer, v. 12, n.1, p. 45-53, 2005. Disponível em: $<$ http://geography.uwo.ca>

BIMBO, F.; BONANNO, A.; NARDONE, G.; VISCECCHIA, R. The Hidden Benefits of Short Food Supply Chains: Farmers' Markets Density and Body Mass Index in Italy. International Food and Agribusiness Management Review, v.18, n.1, p. 1, 2015. Disponível em: <http://ageconsearch.umn.edu>

BOREC, A.; PRISENK, J. Models of Partnerships And Organisational Forms In Short Food Supply Chains In The Slovenian Mountains. Economics of Agriculture, v. 60, n. 2, p. 277-286, 2013. Disponível em: <https://dx.doi.org/339.13:631.115.11(497.4)>. doi: 339.13:631.115.11(497.4)

CROWE, J.; SMITH, J. The influence of community capital toward a community's capacity to respond to food insecurity. Community Development, v. 43, n. 2, p. 
169-186, 2012. Disponível em: <http://dx.doi.org/10.1080/15575330.2011.575230>. doi: $10.1080 / 15575330.2011 .575230$

DONALD, B.; GERTLER, M.; GRAY, M.; LOBAO, L. Re-regionalising the food system?, Cambridge Journal of Regions, Economy and Society, v. 3, n. 2, p.171175, 2010. Disponível em: <http://dx.doi.org/10.1093/cjres/rsq020>. doi: 10.1093/cjres/rsq020

FEENSTRA, G. Creating space for sustainable food systems: lessons from the field, Agriculture and Human Values, v. 19, n. 2, p. 99-106, 2002. Disponível em: <http://hdl.handle.net/10.1023/A:1016095421310>. doi: 10.1023/A:1016095421310

FORSSELL, S; LANKOSKI, L. The sustainability promise of alternative food networks: an examination through "alternative" characteristics. Agriculture and human values, v. 32 , n. 1, p. 63-75, 2015. Disponível em: <https://doi.org/10.1007/s10460-014-9516-4>. doi: 10.1007/s10460-014-9516-4

HAAS, R.; STERNS, J.; MEIXNER, O.; NYOB, D.I.; TRAAR, V. Do US consumers Perceive Local and Organic Food Differently? An Analysis Based on Means-end chain Analysis and Word Association. International Journal on Food System Dynamics, v. 4, n. 3, p. 214-226, 2014. Disponível em: <http://dx.doi.org/10.18461/ijfsd.v4i3.435>. doi:10.18461/ijfsd.v4i3.435

HINRICHS, C. Embeddedness and local food systems: notes on two types of direct agricultural market. Journal of Rural Studies v. 16, p. 295-303, 2000. Disponível em: $\quad<$ https://doi.org/10.1016/S0743-0167(99)00063-7>. doi: 10.1016/S07430167(99)00063-7

HOLLOWAY L., C. R.; VENN, L.; KNEAFSEY, M.; DOWLER, E.; TUOMAINEN, H Managing sustainable farmed landscape through 'alternative' food networks: A case study from Italy. Geographical Journal v. 172, n. 3, p. 219-229, 2006. Disponível em: <http://dx.doi.org/10.1111/j.1475-4959.2006.00205.x> doi: 10.1111/j.14754959.2006.00205.x

HUGHES, D. W.; ISENGILDINA-MASSA, O. The economic impact of farmers' markets and a state level locally grown campaign. Food Policy, v. 54, p. 78-84, 2015. Disponível em: http://dx.doi.org/10.1016/j.foodpol.2015.05.001>. doi: 10.1016/j.foodpol.2015.05.001

ILBERY, B. et al. Forecasting food supply chain developments in lagging rural regions: evidence from the UK. Journal of Rural Studies, v. 20, n. 3, p. 331-344, 2004. Disponível em: <https://doi.org/10.1016/j.jrurstud.2003.09.001>. doi: 10.1016/j.jrurstud.2003.09.001

ILBERY, B.; MAYE, D. Food supply chains and sustainability: evidence from specialist food producers in the Scottish/English borders. Land Use Policy, v. 22, n. 4, p.331-344, 2005. Disponível em: <http://dx.doi.org/10.1016/j.landusepol.2004.06.002>. doi: 10.1016/j.landusepol.2004.06.002

KIRWAN, J. Alternative strategies in the UK agro-food system: interrogating the ENCICLOPÉDIA BIOSFERA, Centro Científico Conhecer - Goiânia, v.14 n.25; p. 286 
alterity of farmers' markets, Sociologia Ruralis, v. 44, n. 4, p. 395-415, 2004. Disponível em: <http://dx.doi.org/10.1111/j.1467-9523.2004.00283.x>. doi: 10.1111/j.1467-9523.2004.00283.x

LEHTINEN, U. Sustainability and local food procurement: a case study of Finnish public catering, British Food Journal, v. 114, n. 8, p. 1053 - 1071, 2012. Disponível em: $\quad<h t t p: / / d x . d o i . o r g / 10.1108 / 00070701211252048>$. doi:

10.1108/00070701211252048

LEVIDOW, L.; PSARIKIDOU, K Food relocalization for environmental sustainability in Cumbria. Sustainability, v. 3, p. 629-719, 2011. Disponível em: <http://doi.org/10.3390/su3040692>. doi: 10.3390/su3040692

LIM, K. H.; HU, W. How local is local? A reflection on Canadian local food labeling policy from consumer preference. Canadian Journal of Agricultural Economics/Revue canadienne d'agroeconomie, v. 64, n. 1, p. 71-88, 2016. Disponível em: <http://dx.doi.org/10.1111/cjag.12062> doi: 10.1111/cjag.12062

MARSDEN, T.; BANKS, J.; BRISTOW, G. Food supply chain approaches: Exploring their role in rural development. Sociologia Ruralis, v. 40, n. 7, p. 424-438, 2000. Disponível em: <http://dx.doi.org/10.1111/1467-9523.00158>. doi: 10.1111/14679523.00158

MASTRONARDI, L.; MARINO, D.; CAVALLO, C, A.; GIANNELLI, A. Exploring the Role of Farmers in Short Food Supply Chains: The Case of Italy. International Food and Agribusiness Management Review, v. 18, n. 2, p. 109, 2015. Disponível em: <http://dx.doi.org/10.1108/BFJ-04-2015-0168>. doi: 10.1108/BFJ-04-2015-0168

MORGAN, K.; MURDOCH J. Organic vs. conventional agriculture: Knowledge, power and innovation in the food chain. Geoforum, v. 3, n. 2, p. 159-173, 2000. Disponível em: <http://dx.doi.org/10.1016/S0016-7185(99)00029-9>. doi: 10.1016/S00167185(99)00029-9

MIGLIORE, G.; SCHIFANI, G.; CEMBALO, L. Opening the black box of food quality in the short supply chain: Effects of conventions of quality on consumer choice. Food Quality and Preference, v. 39, p. 141-146, 2015. Disponível em: $<$ http://dx.doi.org/10.1016/j.foodqual.2014.07.006>.

doi: 10.1016/j.foodqual.2014.07.006

RENTING, H.; MARSDEN, T.; BANKS, J. Understanding alternative food networks: exploring therole of short food supply chains in rural development Environment and Planning A, v. 35, n. 3, p. 393-411, 2003. Disponível em: <http://dx.doi.org/10.1068/a3510>. doi: 10.1068/a3510

ROCKSTRÖM, J.; STEFFEN, W.; NOONE, K. et al. A safe operating space for humanity. Nature, v. 461, n. 7263 , p. 472-475, 2009. Disponível em: <http://dx.doi.org/10.1038/461472a>. doi: 10.1038/461472a

ROSS, N. How civic is it? Success stories in locally focused agriculture in Maine. Renewable Agriculture and Food Systems, v, 21, n. 2, p. 114-123, 2005. 
Disponível em: <https://doi.org/10.1079/RAF2005134>. doi: 10.1079/RAF2005134

ROGERS, J.; FRASZCZAK, M. 'Like the Stem Connecting the Cherry to the Tree': The Uncomfortable Place of Intermediaries in a Local Organic Food Chain. Sociologia ruralis, v. 54, n. 3, p. 321-340, 2014. Disponível em: <http://dx.doi.org/doi/10.1111/soru.12041>. doi: 10.1111/soru.12041

SONNINO, R.; MARSDEN, T. Beyond the divide: Rethinking relations between alternative and conventional food networks in Europe. Journal of Economic Geography, v. 6, p. 181-199, 2006. Disponível em: <https://doi.org/10.1093/jeg/lbi006>. doi: 10.1093/jeg//bi006

SMITH, K.; LAWRENCE, G.; MACMAHON, A.; MULLER, J.; BRADY, M. The resilience of long and short food chains: a case study of flooding in Queensland, Australia. Agriculture and Human Values, v. 33, n. 1, p. 1-16, 2015. Disponível em: <http://dx.doi.or/10.1007/s10460-015-9603-1>. doi: 10.1007/s10460-015-9603-1

TUDISCA, S.; DI TRAPANI, A. M.; SGROI, F.; TESTA, R. Socio-economic assessment of direct sales in Sicilian farms. Italian Journal of Food Science, v. 27, n.1, p. 1K, 2015. Disponível em: <http://dx.doi.org/10.14674/1120-1770\%2Fijfs.v80>. doi: $10.14674 / 1120-1770 \% 2 F i j f s . v 80$

WUBBEN, E.; FONDSE, M.; PASCUCCI, S. The importance of stakeholder-initiatives for business models in short food supply chains: the case of the Netherlands. Journal on Chain and Network science, v. 13, n. 2, p. 139-149, 2013. Disponível em: <http://dx.doi.org/10.3920/JCNS2013.1004>. doi: 10.3920/JCNS2013.1004 